BACTERIAL PATHOGENESIS

\title{
Hitching a ride with DCs
}

Most lineages of Salmonella enterica subsp. enterica serovar Typhimurium cause gastroenteritis in humans, but a recently emerged lineage that was found in sub-Saharan Africa causes systemic disease. Carden et al. now report that the hyper-dissemination of this lineage arises as the result of pseudogenization of a bacterial effector protein that usually inhibits the migration of dendritic cells (DCs).

Invasive $S$. Typhimurium infections in Africa are generally caused by isolates of sequence type 313 (ST313), whereas gastroenteritisinducing strains are usually ST19 isolates. Compared with ST19

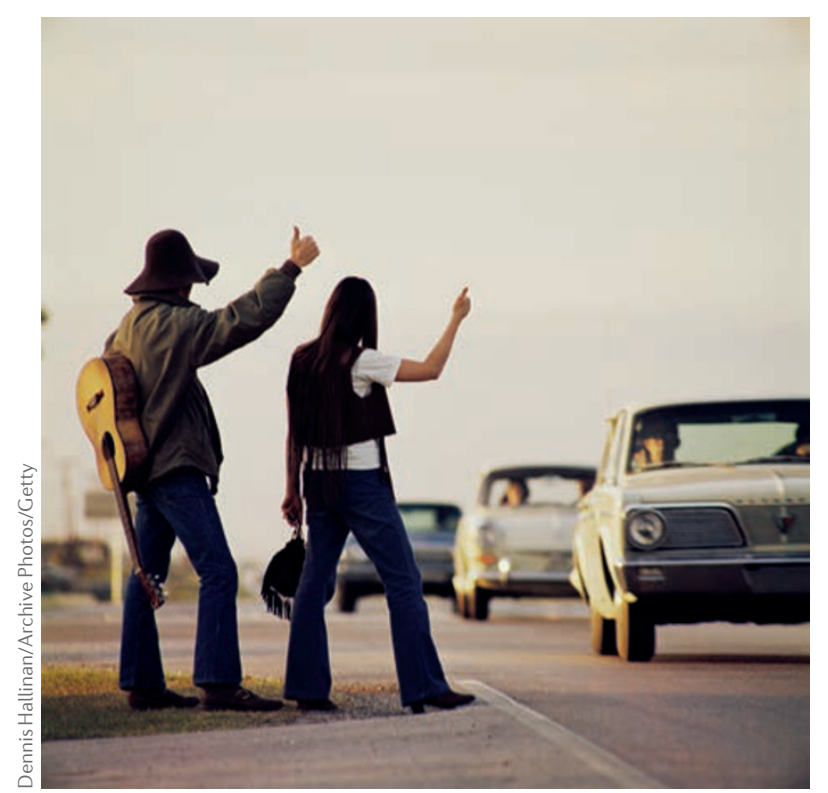

isolates, ST313 isolates show evidence of genome degradation, including the formation of pseudogenes.

When antibiotic-treated mice were orally infected with either ST313 or ST19 isolates of S. Typhimurium, comparable levels of each isolate were detected in the small intestine, Peyer's patches and the caecum 2 days post-infection. By contrast, higher levels of ST313 were detected in the mesenteric lymph nodes (MLNs), spleen and the liver. Mice that were infected intraperitoneally showed similar levels of ST313 and ST19 bacteria in the spleen and the liver, which suggests that the ST313 isolates do not have a survival or replicative advantage at systemic sites, but instead are more efficient at disseminating from the gut.

In mice that had been infected orally with ST19 or ST313 isolates, similar bacterial loads were detected in the blood between 30 minutes and 2 days post-infection; therefore, the initial hyper-dissemination of ST313 from the gut does not occur through the bloodstream. Flow cytometry analyses of innate immune cells in the MLNs showed that ST313 infected more migratory DCs than ST19, which indicates that DCs may facilitate increased dissemination. ST313 was not more efficient in infecting or surviving in DCs than ST19. However, when the authors orally infected mice that were deficient in the lymph node homing receptor CCR7 with either ST19 or ST313, they found similar levels of both isolates in the MLNs, the liver and the spleen. Therefore, CCR7dependent migration of migratory DCs seems to be necessary for the hyper-dissemination of ST313.

Previous work by the same group showed that ST19 produces seven Salmonella pathogenicity island 2 (SPI-2) effectors that inhibit the migration of DCs. One such SPI-2 effector, Salmonella-secreted effector I (SseI), has been rendered a pseudogene in ST313 isolates; the authors reasoned that this may permit DCs that are infected with ST313 to more readily migrate to MLNs. In support of this idea, SseI-deficient strains of $S$. Typhimurium showed increased dissemination to MLNs compared with a wild-type strain. The authors propose that the rapid dissemination of $S$. Typhimurium may lead to faster induction of $\mathrm{T}$ cell responses and enhanced pathogen clearance in immunocompetent hosts. By contrast, in immunocompromised hosts, such as those often found in sub-Saharan Africa, hyper-dissemination is less likely to be deleterious for $S$. Typhimurium, potentially facilitating the loss of SseI and evolution towards causing systemic disease.

Yvonne Bordon, Senior Editor Nature Reviews Immunology

This article is modified from the original in Nat. Rev. Immunol. (http://dx.doi.org/10.1038/nri.2017.16)

ORIGINAL ARTICLE Carden, S. E. et al. Pseudogenization of the secreted effector gene ssel confers rapid systemic dissemination of S. Typhimurium ST313 within migratory dendritic cells. Cell Host Microbe http://dx.doi.org/10.1016/ j.chom.2017.01.009 (2017) 